\title{
Nonlinguistic Factors that Affect the Degree of Foreign Accent in Second Language Mandarin
}

\author{
Lani Freeborn ${ }^{1}$, John Rogers ${ }^{2}$ \\ The Chinese University of Hong Kong ${ }^{1}$ \\ The Education University of Hong Kong ${ }^{2}$
}

\begin{abstract}
Previous research findings have established that a number of nonlinguistic factors can influence the strength of perceived foreign accent in second language (L2) speech. However, the majority of past studies have predominantly considered foreign accent of Indo-European languages, notably English. Therefore, it remains unknown whether the same factors influence foreign accent in other languages, such as Mandarin. This article reports findings from a study on nonlinguistic factors affecting the degree of foreign accent in Mandarin as an L2. Seventy L2 learners of Mandarin Chinese recorded speech samples and completed language background questionnaires. Speech samples were rated by 15 native Mandarin speakers for the degree of foreign accent on a 9-point Likert scale. Stepwise multiple regression analysis resulted in a 3-predictor model of pronunciation accuracy: self-rating of foreign accent, Hànyǔ Shuǐpíng Kăoshì (HSK) proficiency level, and motivational reasons. Results suggest that (1) foreign accent in L2 Mandarin may not be affected by the same factors as in previous L2 accent studies and (2) the concepts of accentedness and comprehensibility may be more intricately linked in lexical tone languages such as Mandarin, in comparison to nontonal languages. These findings have wider implications for the field of L2 acquisition, which is dominated by studies of L2 English.
\end{abstract}

\section{Keywords}

foreign accent, second language, Mandarin Chinese, comprehensibility

Studies in Chinese Linguistics, Volume 40, Number 1, 2019, 75-100 DOI: 10.2478/scl-2019-0003 (C2019 by T.T. Ng Chinese Language Research Centre, Institute of Chinese Studies, The Chinese University of Hong Kong 


\section{Introduction}

Foreign-accented speech has previously received a substantial amount of attention within the field of second language acquisition (SLA). Foreign accent is typically defined as speech that differs from native speaker (NS) norms (Munro and Derwing 1998). Research findings from second language (L2) accent studies to date have found that a number of nonlinguistic individual factors can influence the degree of L2 accent such as age of onset (AO), length of residence (LOR) in the target language country, and amount of L2 use. However, research methodologies in these previous studies have varied considerably (Jesney 2004) and have consisted mostly of L2 English learners (Piske et al. 2001). Therefore, it remains unknown whether the same individual factors consistently affect the degree of foreign accent in other L2s. The past decade has seen a renewed interest in L2 pronunciation (Levins 2016). This renewed interest has been accompanied by a shift in research toward linguistic correlates of foreign-accented speech and comprehensibility. Comprehensibility is defined as what is understood by the listener and is not considered equivalent to accentedness (Derwing and Munro 1997). For example, an L2 learner's speech could have a strong foreign accent but still be highly comprehensible. Yet, little is known about the concepts of accentedness and comprehensibility in other L2s, particularly in tonal languages such as Mandarin. New L2 Chinese research does suggest that accentedness and comprehensibility may be intricately linked in Mandarin (Yang 2016), in contrast to previous L2 accent studies' findings. This initial finding means that we are yet to ascertain whether theories and concepts in SLA can be successfully applied across all L2s. In a review of research on L2 Chinese acquisition, Zhao (2011: 568) addressed the need "to explore problems in L2 Chinese acquisition within the framework of SLA research in general." Similarly, Bergeron and Trofimovich (2017) also called for researchers to "extend their current work to L2s other than English". The present study therefore intends to address this gap, by exploring foreign accent in L2 Mandarin.

\section{Background}

\subsection{Accentedness vs comprehensibility}

Learners of an L2 can often be distinguished from NSs by the degree of their foreign accent. Foreign accent is typically defined as "the extent to which an L2 learner's speech is perceived to differ from native speaker (NS) norms" (Munro and Derwing 1998: 160). However, foreign accent does not always equal a lack of intelligibility or comprehensibility. In the literature, intelligibility and comprehensibility are defined as two distinct constructs. Intelligibility refers to what is understood by the listener and is typically measured by listeners' transcriptions of speech samples, whereas comprehensibility refers to listeners' perception of intelligibility and is normally measured using a Likert or sliding scale. When 
investigating the relationship between the three concepts, Derwing and Munro (1997: 15) found that accent, intelligibility, and comprehensibility "are clearly related, but are not equivalent." Several studies have established that it is possible for an L2 learner's speech to be heavily accented and still be understood (Munro and Derwing 1999).

More recently, researchers have aimed to uncover which linguistic aspects of L2 speech are linked to accent and comprehensibility. Findings to date indicate that whereas comprehensibility is tied to a number of factors including grammar, lexis, discourse, and phonological features, accent is linked predominantly to phonological features, in particular, segmental and suprasegmental features (Kang 2010; Saito et al. 2015, Saito et al. 2016; Sereno et al. 2016; Trofimovich and Isaacs 2012). Bergeron and Trofimovich (2017: 561) also found the same results for the speech of L2 French learners, where "accentedness was uniquely associated with the phonology and fluency dimension of L2 speech while comprehensibility was broadly linked to multiple dimensions." Distinguishing among linguistic factors affecting accentedness and comprehensibility is a fairly new line of research in L2 acquisition. The majority of L2 accent studies to date have focused on the roles of nonlinguistic individual factors such as AO, LOR, L2 use, and motivation. The following section focuses on these individual factors and describes the key findings of these studies.

\subsection{Factors affecting the degree of foreign accent}

The importance of learning an L2 from a young age is reflected in the critical period hypothesis $(\mathrm{CPH})$, which claims that language learning becomes more challenging after a certain age due to loss of neural "plasticity" (MacKay et al. 2006; Penfield and Roberts 1959). The CPH is supported by a considerable amount of research on the relationship between AO and the degree of L2 accent (Jesney 2004) in both adult and child L2 learners. With the exception of late L2 learners who achieve native-like proficiency even with AO in adulthood (Moyer 1999), $\mathrm{AO}$ has been found to be a consistent predictor of L2 accent (Flege et al. 1995; Uzal et al. 2015).

LOR in the target language country is another factor which is often linked to the degree of L2 accent but on which previous studies have mixed results. For example, Major (1986) found that participants with the highest (most nativelike) accent scores had all spent at least 1 year living in the target language country, while in contrast, Derwing and Munro's (2013) longitudinal study on the development of L2 oral skills showed that the number of years spent in the target language country had no significant relationship with accent ratings. These equivocal research findings suggest that the degree of L2 accent does not necessarily diminish as LOR increases. Much like AO, some research has shown that LOR may only be a significant predictor of foreign accent when combined with L2 input. In Moyer's (2013: 73) opinion, “(w)here LOR does show main effects, it is arguably the quality of that residence that matters." Purcell and Suter 
(1980) and Flege and Liu (2001) found that LOR only had a significant effect on pronunciation accuracy when combined with the number of months residing with (in the same home as) NSs. This suggests that L2 phonological success depends on input from NSs.

L2 input is unquestionably an important factor affecting both ultimate L2 attainment and degree of accent; as Saito (2015: 564) explained, there is "a general consensus among researchers in the field of SLA that L2 learners' developing system is enhanced as they increase their amount of relevant L2 experience through intensive exposure to the L2." "Relevant L2 experience" here refers to social interaction outside the classroom, in particular with NSs, and its importance is supported by numerous L2 accent studies (Kinsella and Singleton 2014; Suter 1976; Wayland 1997). For example, in a 7-year study, Derwing and Munro (2013) found that having regular conversations with NSs affected comprehensibility scores, but not accentedness. However, in their study, many participants reported having strong ties to their first language (L1) community and continued to frequently speak their L1. In fact, as the following section discusses, the amount of L1 use, separate from L2 use, has also been found to affect the degree of foreign accent.

Research findings suggest that even if learners have a large amount of relevant L2 experience and exposure, they is still likely to have a notably foreign accent if they continues to use their L1 often. Several studies of native Italians living in Canada have linked the degree of foreign accent with more L1 use (Flege et al. 1997; Flege et al. 1995), for both early and late bilinguals (Piske et al. 2001), and the same effect has also been found for other language combinations, for both adults and children (Guion et al. 2000; Uzal et al. 2015). However, few previous studies have examined whether speakers' L1 background, regardless of L1 use, affects foreign accent.

The role of motivation has received a great deal of attention in SLA research. A number of studies support Moyer's (2016: 21) view that "( $\mathrm{t}$ )here is no question that attitudes drive language acquisition and are especially influential for L2 pronunciation." One study that attempted to directly measure the effects of motivation on L2 accent attainment was conducted by Polat and Schallert (2013) on Kurdish adolescents acquiring Turkish, which found that "degree of identification with the Turkish-speaking community was a positive predictor of a native-like accent" (Polat and Schallert 2013: 755). Similarly, Kinsella and Singleton (2014) found that late L2 French learners with the highest test results intended to reside in France indefinitely. Another motivational factor is speakers' concern with pronunciation. Suter (1976) and Purcell and Suter (1980) found that the strength of concern for pronunciation accuracy was one of the most significant variables affecting degree of foreign accent. In contrast, in the study by Moyer (1999), concern with pronunciation was not a significant factor, but professional motivation made up $41 \%$ of the variance. These studies suggest that motivational 
factors do play at least some role in determining the strength of a speaker's foreign accent, although more research is needed to establish just how important motivation is in L2 accent attainment.

In addition to the individual factors discussed so far, the degree of L2 accent may also depend on natural ability or aptitude. L2 accent studies typically measure aptitude through oral mimicry tests, which assess participants' ability to mimic speech sounds in the L2; mimicry ability has been found to affect the degree of L2 accent in several studies (Thompson 1991; Purcell and Suter 1980). In a review of L2 accent studies, Piske et al. (2001: 202) noted that the "ability to mimic unfamiliar speech sounds ... has repeatedly been identified as a significant and independent predictor of degree of L2 foreign accent." Oral mimicry ability has also been empirically linked to musical ability, based on the idea that both music and language draw on suprasegmentals such as intonation, rhythm, pitch, and stress (Zybert and Stepien, 2009). Several studies have confirmed a positive relationship between speakers' musical ability and L2 phonological ability (Slevc and Miyake 2006; Zybert and Stepien 2009). Furthermore, researchers have linked musical ability specifically to the learning of L2 tones, as are present in Mandarin. For example, Bowles et al. (2016) found that participants' musicality and pitch ability better predicted the learning of L 2 tones than general cognitive ability and aptitude. There are some additional factors that may affect the degree of foreign accent but have garnered mixed findings or been less researched than others. These include sex (Flege et al. 1995; Thompson 1991) and amount of instruction (Derwing and Munro 2013; Moyer 1999).

This section has discussed which factors are currently known to affect the degree of foreign accent. It is important to point out that the majority of research into this area has focused on the L2 learning of European languages, in particular English. There is a limited amount of research into foreign accent in tonal languages, and even less research into foreign-accented Chinese.

\subsection{Chinese as L2 research}

As can be seen from previous research, a number of factors have been found to affect the degree of L2 accent. However, it is difficult to draw any overall conclusions from these findings since not all past studies have included the same variables. Further, the scarcity of accent studies in L2s besides English is problematic, because these research findings may or may not be comparable to other languages, such as Mandarin. As Zhao (2011: 566) pointed out, "findings in L2 Chinese studies have presented challenges to L2 theories and models which are based on L2 acquisition of European languages." This may also apply to L2 Mandarin accent studies, especially given that Mandarin is a lexical tone language. As previously discussed, L2 accent studies have ascertained that accentedness and comprehensibility, while related, can be considered as two separate constructs, with raters typically judging the degree of accent by segmental or suprasegmental 
pronunciation errors. However, in lexical tone languages such as Mandarin, pronunciation errors could be more likely than in other languages to result in a lack of comprehension, rather than simply indicating the presence of a foreign accent. This is because in contrast to a language like English, where tone or pitch can be altered to add functional meaning, for example, to add emphasis or emotion, an alteration in tone or pitch in Mandarin changes the lexical meaning of the word. Table 1 lays out the 4 standard Mandarin tones and summarizes how altering the tone and pitch of a Mandarin word can result in a different lexical meaning.

Table 1 The four main Mandarin tones (taken from Sun 2006: 139)

\begin{tabular}{|l|l|l|l|}
\hline Tone 1 & Tone 2 & Tone 3 & Tone 4 \\
\hline Mā 媽 & Má 麻 & Mă 馬 & Mà 罵 \\
\hline
\end{tabular}

The relationship between accentedness and comprehensibility in L2 Mandarin was explored recently in a study by Yang (2016), where the speech samples of 20 American L2 learners of Mandarin were judged for both accentedness and comprehensibility. Yang (2006: 138) found a strong negative correlation between comprehensibility and accent scores and explained that "the effect of prosodic deviations, especially tone errors, on foreign accent ratings, is represented via comprehension." Listeners were also asked to note the criteria they used to judge foreign accentedness; their notes revealed that, in addition to prosodic features such as tone and breaks, listeners rated foreign accent according to how well they understood the speaker. These results suggest that the constructs of accentedness and comprehensibility may be more difficult to disentangle in L2 Mandarin compared to nontonal L2s; however, Yang is the only researcher to date to have investigated accentedness and comprehensibility of L2 Mandarin, and little is known about foreign accent in tonal languages in general.

Previous research into L2 Mandarin phonology has largely focused on the perception of tone errors and the effects of having a tonal or nontonal L1 (e.g., Godfroid et al. 2017). For example, in the study by So and Best (2010), L1 Cantonese and Japanese speakers (both of which represent tonal L1s) outperformed L1 English speakers when identifying L2 Mandarin tones. However, they also found that the Cantonese group confused tone 2 (T2) and tone 3 (T3), which may be because there is no T3 equivalent in Cantonese. Similarly, So and Best (2014) found that although L1 French speakers identified tones more accurately than L1 English speakers, both groups had difficulties distinguishing between T2 and T3 as well as tone 1 (T1) and tone 4 (T4). In order to test the influence of L1 lexical tone experience on L2 Mandarin tone learning, Wang (2013) included L1 speakers of Hmong (a lexical tone language), Japanese, and English. The Japanese and English were significantly 
more accurate in tone perception than the Hmong group, although all groups made gains in a posttest. In contrast, Hao (2012) found no significant overall effect of L1 when measuring L1 English and Cantonese learners' perception of L2 Mandarin tones. These mixed results suggest that speakers of tonal L1s have neither overall advantage nor disadvantage over speakers of nontonal L1s in the perception of L2 Mandarin tones. Less research has focused on the production of L2 Mandarin tones, although some studies have set out to investigate the relationship between perception and production. For example, Bei (2012) found that L2 Mandarin learners' tonal production was better than their tonal perception. In another recent study, Hao (2018) noted some inconsistencies between learners' perception and production, particularly regarding T3 and the prosodic position of surrounding tones.

To date, research into L2 Mandarin has only focused on how linguistic factors, such as tone errors and L1 effects, influence the perception and production of individual tones. There is a lack of research concerning nonlinguistic factors that affect the degree of foreign accent in L2 Mandarin.

The present study aims to address this gap by asking the following research question:

RQ1: Which nonlinguistic factors affect the degree of foreign accent in L2 Mandarin?

\section{Methods}

\subsection{Participants}

\subsubsection{Speakers}

In all, 70 speakers, students at universities in Nanjing, China, were recruited to participate in this study (39 female, 31 male). Their characteristics are reported in Table 2. The mean age of speakers was 23.14 years $(\mathrm{SD}=3.08)$ with a range of 19 33 years. Some had been in Nanjing for several years and were doing undergraduate or postgraduate degrees in China, whereas others had come to study for just 1 or 2 semesters. Therefore, their LOR ranged greatly, from 2 months to 7 years, with a mean of 19 months. The amount of formal instruction in Mandarin they had received also varied considerably, ranging from 4 months to 10 years, with a mean of 3 years. Speakers came from 19 different first-language backgrounds (14 English, 13 French, 6 German, 5 Korean, 5 Russian, 4 Arabic, 4 Spanish, 3 Dutch, 3 Ukrainian, 2 Darija, 2 Mauritian Creole, 2 Hebrew, 1 Czech, 1 Estonian, 1 Kinyarwanda, 1 Polish, 1 Swedish, 1 Tongan, and 1 Urdu). All speakers spoke English to at least intermediate level in addition to their L1 and their L2 Mandarin. In order to understand the material used in the study, speakers were required to have a minimum of Hànyŭ Shuǐpíng Kăoshì (HSK) (Chinese Proficiency Test) level 4, which corresponds approximately to B2 (approximately upper-intermediate level) in the Common European Framework of Reference (CEFR) for languages. 
Speakers who did not know their HSK level were asked to review the reading text and were accepted to the study if they were familiar with and understood all of the characters. Speech samples of 4 native Mandarin speakers (2 female, 2 male) were also included in the study, to verify listeners' ability to identify native speech. All participants gave informed consent.

Table 2 Mean characteristics of the 70 speaker participants

\begin{tabular}{|l|c|c|c|}
\hline Variable & Mean & SD & Range \\
\hline Chronological age & 23.14 & 3.08 & $19-33$ \\
\hline AO & 19.73 & 3.43 & $13-31$ \\
\hline L1 use (\%) & 39.03 & 20.49 & $0-80$ \\
\hline L2 use (\%) & 29.79 & 11.84 & $10-60$ \\
\hline Amount of formal Mandarin instruction (in months) & 34.10 & 24.78 & $4-120$ \\
\hline LOR (in months) & 18.99 & 18.46 & $2-96$ \\
\hline
\end{tabular}

Abbreviations: AO, age of onset; LOR, length of residence; L1, first language; L2, second language.

\subsubsection{Listeners}

Speech samples were rated by 15 listeners (12 female, 3 male) who were NSs of Mandarin, all from Nanjing, China. Their mean age was 23.73 years (SD = 7.04), range 18-42 years. To control for possible effects of listener familiarity and to match the speakers (who all spoke English in addition to their L1 and Mandarin), all listeners had at least pre-intermediate-level English. None reported having above elementary ability in another L2. Five listeners had spent time abroad in Australia, and the other 10 had not spent any time in a foreign country. All listeners reported normal hearing ability.

\subsection{Materials}

Speakers read a paragraph of text (Appendix A) including both Chinese characters and pinyin (Chinese written in the Roman alphabet). The text was selected, with the help of a native Mandarin teacher, from HSK material levels 3-4. HSK is the only standardized Chinese language proficiency test for non-NSs (NNSs) and consists of 6 levels, which correspond approximately to the CEFR for languages. In the current study, the Chinese learners read speech out loud rather than producing extemporaneous speech, because prior researchers have shown that accent ratings can be affected by grammatical and lexical errors (Major 1986). Recordings took place in quiet locations around university campuses, using a Tsinghua Tongfang digital audio recorder. Speakers were given a few minutes to familiarize themselves with the text before recording. Speakers completed the language background questionnaire prior to recording; it was adapted from the Language Experience and Proficiency Questionnaire (LEAP-Q; Marian et al. 
2007). The total time for each speaker to complete both the questionnaire and the recording was 15-20 minutes. The 74 speech samples (70 NNSs and 4 NSs) were edited using the software Audacity, with only the first two sentences of each sample used for ratings. Because researchers investigating L2 accent have uncovered task-order effects in their studies (Jesney 2004), we put the samples onto $3 \mathrm{CDs}$, each randomized in a different order.

\subsection{Rating procedure}

Rating took place in a quiet room at the first author's school, in three sessions, each with a different group of listeners and a different randomized CD. Listeners rated speech samples for the degree of foreign accent, on a 9-point Likert-type scale $(1=$ very strong foreign accent, $9=$ no foreign accent). In keeping with previous L2 accent studies, speech samples were heard once, no training was provided, and listeners were instructed to use the whole length of the scale. Listeners were instructed to only give a rating of 9 (no foreign accent) if they judged the speaker as a native Mandarin speaker. All listeners correctly identified the 4 native Mandarin speech samples. They had two 3-minute breaks during the rating session to reduce fatigue; they were instructed not to discuss their judgments with other listeners. Three practice samples were given prior to rating (1 low, 1 medium, 1 high), selected with the assistance of a native Mandarin teacher. These samples served to familiarize listeners with the task and were not included in the analyses. Each rating session lasted 25-35 minutes.

\subsection{Data analysis}

\subsubsection{Independent variables}

Information collected from the language background questionnaires was used to create a dataset using IBM SPSS Statistics 22, for 17 independent variables:

- Age: chronological age in years.

- $\quad$ Sex: female coded as 1 , male coded as 2 .

- L1: speakers listed all languages spoken, in order both of acquisition and of dominance. Because there were 19 different L1s, some L1s were grouped together, and so a total of $6 \mathrm{~L} 1$ groups were included for this variable. L1 groups were dummy coded $(1=$ French $[\mathrm{n}=13], 2=$ German and Dutch $[\mathrm{n}=9], 3=$ English [ $\mathrm{n}=14], 4=$ Russian, Ukrainian, Polish, and Czech $[\mathrm{n}=10], 5=$ Arabic and Darija $[\mathrm{n}=6], 6=$ Korean $[\mathrm{n}=5])$. Other L1s (e.g., Tongan $[\mathrm{n}=1]$ and Urdu $[\mathrm{n}=1]$ ) were entered as missing data and not included in the analyses.

- Additional languages spoken: number of languages participants spoke in addition to their L1 and Mandarin.

- Formal musical training: number of years of formal musical training, including vocal training. Multiple instruments were counted separately (e.g., piano: 3 years; guitar: 2 years; total: 5 years). 
- L1 use: current L1 use as a percentage of total language use, to the nearest $10 \%$.

- $\quad$ L2 use: percentage of current L2 use to the nearest $10 \%$.

- AO: age when first began learning Mandarin.

- Length of instruction: amount of formal Mandarin instruction received (data entered in the number of months).

- $\quad$ LOR: time spent in a Mandarin-speaking country (in months).

- $\quad$ HSK level: current HSK level. Levels range from 1 to 6; the minimum level required for the study was level 3. If participants had not taken the HSK examination and/or were unsure of their current level, data were entered as missing.

- $\quad$ Socialize: How often their time spent socializing consisted of socializing in Mandarin with native Mandarin speakers on a scale of 1 (never) to 4 (always)?

- Self-rating: How strong (how thick) they thought their foreign accent was in Mandarin, on the same 9-point Likert scale used in the listener ratings ( $1=$ very strong foreign accent, $9=$ no foreign accent)?

- Importance of accent: How important it was for them to sound like a native Mandarin speaker, from 1 (not important) to 5 (very important)?

- Motivation: Why they were learning Mandarin. They could choose from study, personal interest, and work and could tick all applicable. Data were coded as $1=$ study, $2=$ personal, $3=$ work, $4=$ any 2 reasons, $5=$ all reasons.

- Knowledge of tonal languages: taken from the list of languages spoken, with proficiency levels; coded as $1=$ no knowledge, 2 = elementary, $3=$ intermediate, $4=$ advanced, $5=\mathrm{NS}$.

- $\quad$ Bilingual: ${ }^{1} 0=$ monolingual, $1=$ bilingual.

\subsubsection{Inter-rater reliability}

Cronbach's alpha was calculated to verify the inter-rater reliability of the 15 native Mandarin listeners. Results indicated high internal consistency, with alpha of 0.941. The mean accent rating score for each speaker was then calculated and added to the dataset for subsequent statistical analyses.

\subsubsection{Statistical analyses}

All statistical procedures were carried out using SPSS 22, following the guidelines for stepwise regression analysis of Pallant (2010). The first stage of data analysis was to explore the intercorrelatedness between predictor variables, using Pearson bivariate correlations calculated between all independent variables and mean accent score ratings and interpreted as small (0.25), medium (0.38), or large (0.60), following Plonsky and Oswald (2014). In keeping with previous L2 accent studies (Purcell and Suter 1980; Thompson 1991) and with quantitative L2 research in general (Plonsky 2015), the alpha level was set at 0.05 .

1 Excluding L2 Mandarin. 
The independent variables that showed significant correlations with the dependent variable were then entered into a stepwise multiple regression analysis, in order to ascertain which combination of independent variables best predicted accent ratings. Like correlations, regression analysis is a test of association, not causation. However, regression analysis is preferable to simple correlations, because it is difficult to assess the true effect of independent variable based solely on the latter, particularly if there is a large amount of intercorrelatedness between predictor variables and/or a relatively large number of predictor variables (Tabachnick and Fidell 2013). Stepwise regression is also an advantageous form of data analysis for exploratory studies; as Hosmer and Lemeshow (2000: 116) explained, when "the outcome being studied is relatively new and the important covariates may not be known ... a stepwise selection procedure can provide a fast and effective means to screen a large number of variables." In addition, previous studies in SLA as well as several L2 accent studies have used this procedure (Flege et al. 1995; Purcell and Suter 1980; Thompson 1991), allowing comparability between the current results and past findings.

A forward stepwise regression begins with no predictors in the model; independent variables are added one at a time until the model has found the combination of independent variables with the highest total $R^{2}$ value. Regression results are interpreted based on the $R^{2}$ values, which show the percentage of variance in the dependent variable (here, mean accent ratings) accounted for by the predictor variables. Due to the risk of important variables being excluded from the model, a liberal alpha level of 0.15-0.20 is best (Hosmer and Lemeshow 2000; Tabachnick and Fidell 2013); thus, we selected an alpha of 0.20 for the current study. Prior to conducting the stepwise analysis, care was taken to ensure that the assumptions of the test were met, following criteria outlined by Pallant (2010: 151) regarding multicollinearity and singularity, outliers, normality, linearity, homoscedasticity, and independence of residuals.

\section{Results}

\subsection{Correlations among independent variables}

Several of the predictor variables were highly intercorrelated. Table 3 summarizes the matrix of all Pearson correlation coefficients for 16 independent variables (excluding the categorical variable of the L1 group). The strongest correlation $(0.822)$ is between participants' age and AO. As might be expected, there are also medium-to-large correlations between the HSK level and the amount of formal instruction (0.508) and the HSK level and LOR (0.488). Moreover, unsurprisingly, there is a medium-to-large negative correlation between L1 use percentage and number of additional languages spoken (-0.464). Interestingly, there are negative correlations between LOR and motivational reasons for learning Mandarin $(-0.376)$ as well as a small negative relationship between LOR and the importance of sounding like a native Mandarin speaker (-0.205). 


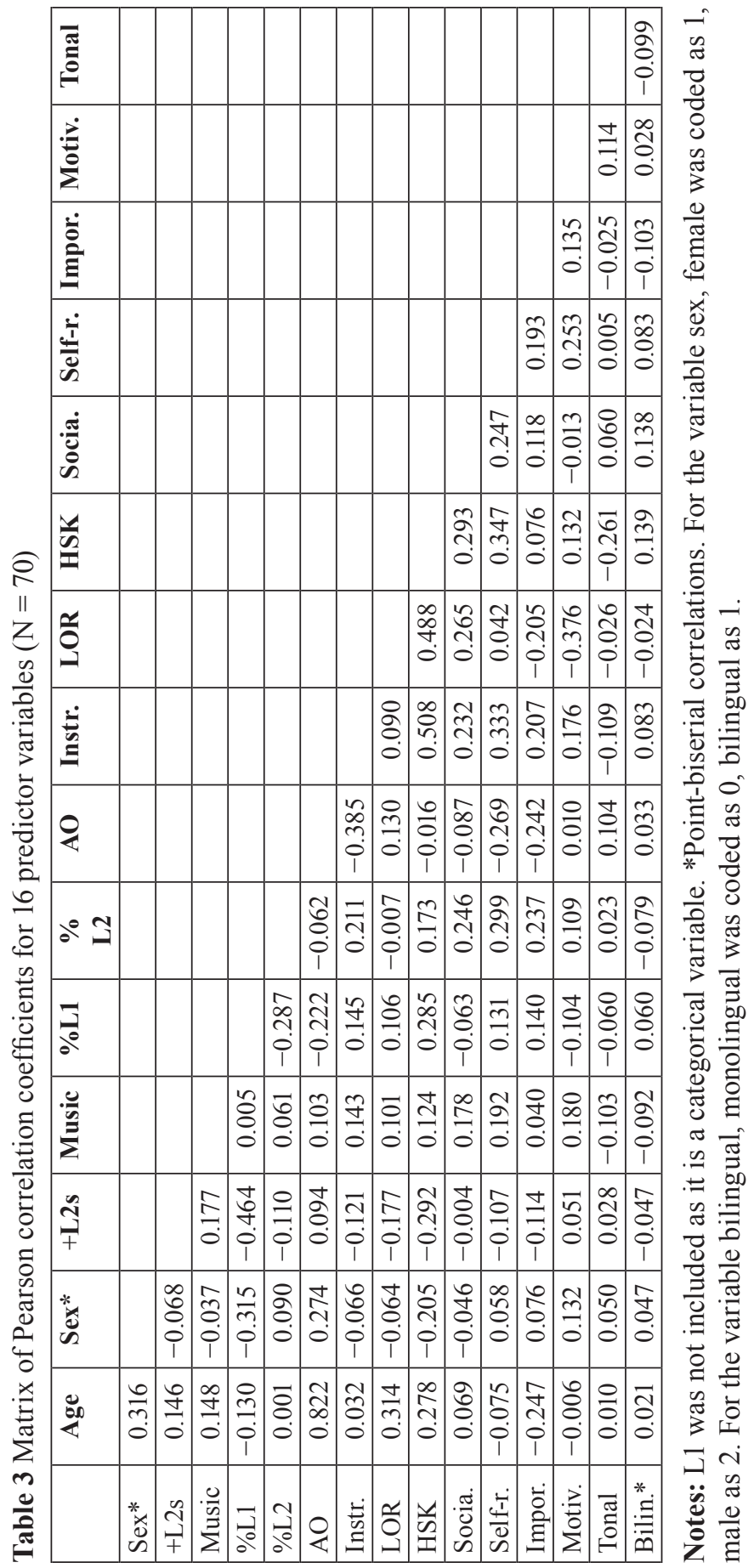

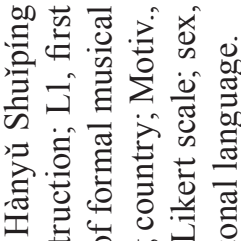

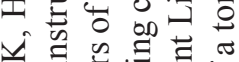

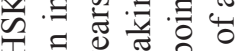
I $\supset$ 웡

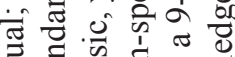

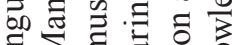
$\Xi \sum_{4}$ के তँ 50 $\Xi \Sigma .00$ : 踏劳 苛 $\infty$ 음 घ1

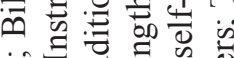
过吾过 ठั

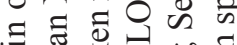

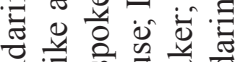

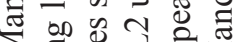
$\sum \equiv$ 品 त

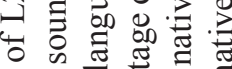

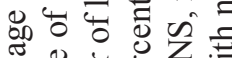
ठ एँ

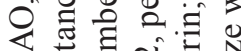
的苛寻䨌 क्षे की ब. जे के

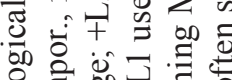
응 要击当

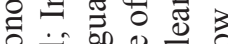

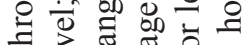
0 元

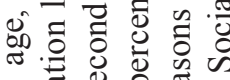
$\ddot{0} 0$ 을 寻元 ज्ञ 들

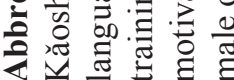




\subsection{Correlations between independent variables and mean accent rating}

The next stage of data analysis was to conduct Pearson bivariate correlations between the independent variables (excluding the L1 group) and mean accent ratings. Table 4 summarizes the correlation coefficient and probability statistic for each of these. Of the 16 predictor variables, 8 showed a significant relationship with mean accent rating. Out of these 8 variables, the strongest correlation with accent ratings was for speakers' self-rating of foreign accent (0.470), followed by HSK proficiency level (0.406) and amount of L2 instruction (0.339). Motivational factors such as reasons for learning Mandarin (0.317) and importance of sounding like an NS (0.317) showed medium correlations and formal musical training $(0.301)$ and amount of L2 use (0.273), weak correlations. Interestingly, being bilingual had a medium negative correlation $(-0.239)$, and LOR, unexpectedly, a small negative correlation $(-0.150)$, implying that the longer speakers' residence in a Mandarinspeaking country, the stronger their foreign accent.

Table 4 Pearson correlation coefficients between mean accent rating scores and independent variables

\begin{tabular}{|l|c|c|}
\hline Independent variables & $\begin{array}{c}\text { Correlation } \\
\text { coefficient }(\boldsymbol{r})\end{array}$ & $\begin{array}{c}\text { Probability } \\
\text { statistic }(\boldsymbol{p})\end{array}$ \\
\hline Age & +0.089 & 0.466 \\
\hline Sex & +0.037 & 0.759 \\
\hline $\begin{array}{l}\text { Number of languages spoken in addition to } \\
\text { L1 and L2 Mandarin }\end{array}$ & -0.075 & 0.537 \\
\hline Music & $+0.301^{*}$ & 0.011 \\
\hline \%L1 use & +0.153 & 0.205 \\
\hline \%L2 use & $+0.273^{*}$ & 0.022 \\
\hline AO & -0.103 & 0.394 \\
\hline Amount of formal Mandarin instruction & $+0.339^{* *}$ & 0.004 \\
\hline LOR & -0.150 & 0.216 \\
\hline HSK proficiency level & $+0.460^{* *}$ & 0.002 \\
\hline How often socialize in Mandarin & +0.135 & 0.264 \\
\hline Self-rating of foreign accent & $+0.470^{* *}$ & 0.000 \\
\hline Importance of sounding like an NS & $+0.317^{* *}$ & 0.007 \\
\hline Motivation & $+0.317^{* *}$ & 0.007 \\
\hline Knowledge of tone languages & +0.079 & 0.516 \\
\hline Bilingual & $-0.239^{*}$ & 0.046 \\
\hline
\end{tabular}

Notes: *Correlation is significant at the 0.05 level (2-tailed). ${ }^{* *}$ Correlation is significant at the 0.01 level (2-tailed).

Abbreviations: AO, age of onset; HSK, Hànyǔ Shuǐpíng Kăoshì; LOR, length of residence; L1, first language; L2, second language; \%L1, percentage of L1 use; $\% \mathrm{~L} 2$, percentage of L2 use; NS, native speaker. 


\subsection{Sex}

There was no difference between mean rating for female speakers (5.3) and male speakers (5.4). However, given that the current study is exploratory and that some previous L2 accent studies have found differences between female and male speakers' accent scores, Pearson correlations were also conducted, with a split dataset for female $(n=39)$ and male $(n=31)$ speakers. Although some predictors showed medium-to-large correlations in both groups, for example, HSK level (female 0.500, male 0.439), there were also marked differences; for example, female speakers' accent self-ratings had a large correlation (0.588), whereas male speakers' only showed a medium correlation (0.344). The biggest difference was in the amount of L2 instruction, which was strongly correlated for females ( 0.510$)$, but no relationship for males (0.024). Female participants' accent scores were also affected more by motivational factors than those of male participants - the importance of sounding like an NS (0.451) and motivational reasons (0.415) showed medium-tolarge correlations for females but only very weak correlations for males.

\section{$4.4 \mathrm{~L} 1$}

For L1, as a categorical variable, alternative methods were needed to explore its effects on the degree of foreign accent. As previously mentioned, given the large number (19) of L1 speakers, L1s were grouped together where possible, resulting in $6 \mathrm{Ll}$ groups covering a total of 57 speakers (where there were only 1 or 2 speakers of an L1, e. g., Tongan, these L1s were not included in this data set). Box plots were created to visualize the range and distribution of accent scores for each L1 group (Figure 1). Native Korean speakers received

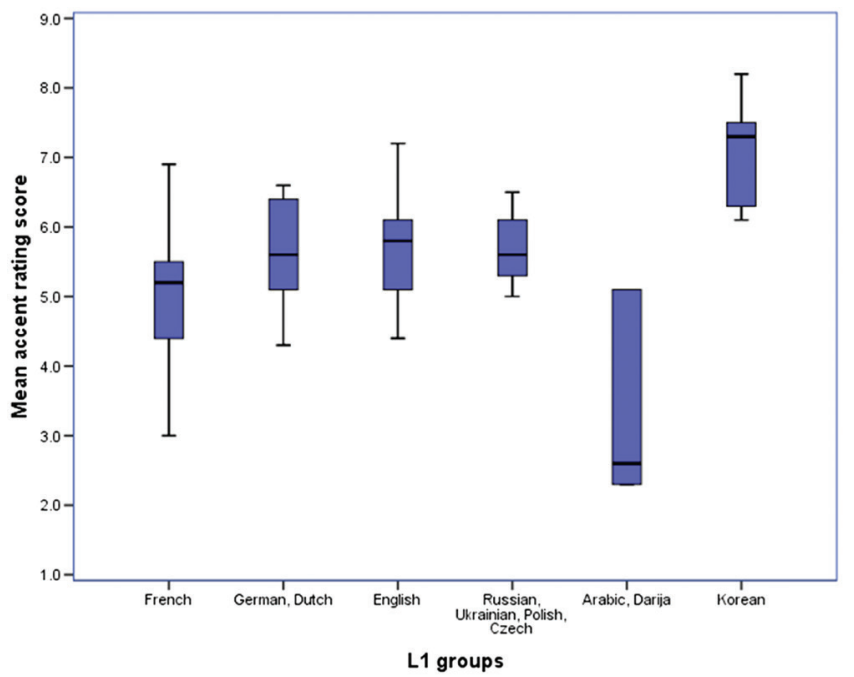

Figure 1 Box plots of the mean accent rating scores for L1 groups Abbreviation: L1, first language. 
the highest accent rating among the L1 groups, with a mean score of 7.0; native Arabic and Darija speakers received the lowest scores, with a mean of 3.3. The mean scores of the other L1 groups were quite similar, although there was variation within them; as the box plots show, native French speakers' scores ranged from 3.0 to $6.9(\mathrm{~m}=5.1)$, whereas speakers of Slavic languages (Russian, Ukrainian, Polish, Czech) had a much smaller range of 5.0-6.5 $(m=5.7)$.

\subsection{Regression analysis}

The 8 independent variables that showed significant correlations with mean accent rating were entered into the stepwise regression analysis. Table 5 summarizes the results of the final regression model, which included 3 variables and accounted for $41 \%$ of the total variance in accent scores. The first variable to enter the regression was self-rating of foreign accent, which accounted for $22 \%$ of the variance; the second was HSK proficiency level, which increased the variance by $10 \%$, followed by the third, motivation, which increased variance by an additional $9 \%$. The overall model was significant, $F(3,83)=8.933, p<$ $0.001, r^{2}=0.41$.

Table 5 Results of the stepwise regression analysis of accent rating scores

\begin{tabular}{|l|l|c|c|c|c|}
\hline Model & Predictors & $\begin{array}{c}\text { Cumulative } \\
\boldsymbol{R}\end{array}$ & $\begin{array}{c}\text { Cumulative } \\
\boldsymbol{R}^{2}\end{array}$ & $\boldsymbol{F}$ & $\boldsymbol{p}$ \\
\hline 1 & $\begin{array}{l}\text { Self-rating of foreign } \\
\text { accent }\end{array}$ & 0.470 & 0.221 & 11.35 & 0.002 \\
\hline 2 & HSK proficiency level & 0.567 & 0.321 & 5.76 & 0.021 \\
\hline 3 & Motivation & 0.637 & 0.405 & 5.38 & 0.026 \\
\hline
\end{tabular}

Abbreviation: HSK, Hànyǔ Shuľpíng Kăoshì.

\section{Discussion}

This study examined which nonlinguistic factors affect the degree of foreign accent in Mandarin as an L2. The correlation results showed that 8 predictor variables were significantly correlated with mean accent rating, and regression analysis resulted in a 3-predictor model including self-rating of foreign accent, HSK proficiency level, and motivation. This section discusses the 3 variables from the regression model as well as the other 5 variables that significantly correlated with mean accent ratings and considers to what extent these results are comparable to existing research on L2 foreign accents.

\subsection{Self-rating of foreign accent}

Self-rating of foreign accent was the strongest correlate (0.470) with mean accent score and was also the first predictor in the regression model. This would suggest that learners of L2 Mandarin are fairly accurate when judging 
the strength of their own foreign accent. A tentative explanation is that in tonal languages, such as Mandarin, accentedness may be more closely linked to comprehensibility than in nontonal languages. This interpretation is supported by Yang (2016), who found a strong negative relationship between accent scores in Mandarin and comprehensibility. Given this fact, the speakers in this study may have gained self-awareness of their accent through their daily interactions in Nanjing and their level of successful or failed communicative interactions. As Yang (2016: 139) explained, accent in L2 Mandarin is critical "as it directly affects comprehension." This view is supported by the positive correlations between self-rating of foreign accent and a number of other independent variables, including HSK proficiency level (0.347), amount of formal Mandarin instruction (0.333), percentage of Mandarin use (0.299), and how often they socialize with native Mandarin speakers $(0.247)$. This combination of higher proficiency level, speaking Mandarin regularly, and socializing with NSs "could" enable speakers to have better self-awareness of their pronunciation, since they would know how often they are understood in the L2. It would appear that the participants in the present study equated their Mandarin foreign accent with comprehensibility.

However, it is difficult to draw firm conclusions regarding this predictor at present, because few previous L2 accent studies have included self-rating of accent as a variable, and the few studies that have, have reported mixed findings. For example, in the studies by Kinsella and Singleton (2014) and Trofimovich et al. (2016), participants' general self-ratings of speech were positively correlated with high, native-like proficiency scores; however, Trofimovich et al. (2016: 13) found "no association between the speakers' actual and self-rated scores for accent." In fact, speakers who received low scores on accent overestimated their ability, whereas speakers who received high scores underestimated their ability. In addition, in a recent study by Jiang and Cohen (2018), learners of L2 Mandarin showed some inconsistencies between their self-reported pronunciation difficulties and their actual pronunciation accuracy.

\subsection{Proficiency level}

The second highest correlation with mean accent rating was that of HSK proficiency level (0.460). Although a number of studies have examined the relationship between proficiency level and comprehensibility and/or intelligibility, to our knowledge, proficiency level has only been included in one L2 accent study. Thompson (1991) found that global speaking proficiency in English had the fourth most significant effect on accent rating out of 15 independent variables. It is important to recognize here that HSK proficiency level should be distinguished from other measures of L2 proficiency due to the design of the HSK speaking tests. Part 1 (of 3) of the HSK test, at all levels (1-6), consists of a "listen and repeat" exercise (China Education Center Ltd. 2016), which tests candidates' oral mimicry ability; thus, test takers need to 
accurately pronounce Mandarin tones to pass this part of the examination. There is an even bigger emphasis on pronunciation accuracy in the HSK advancedlevel speaking test (levels 5 and 6), where candidates are required to do a "read aloud" task in part 2. In fact, two-thirds of the advanced HSK speaking tests focus solely on pronunciation accuracy. Therefore, HSK proficiency level could plausibly reflect participants' amount of pronunciation training and their oral mimicry ability. ${ }^{2}$

In addition, in support of the current findings, past research has shown that pronunciation training can have a positive effect on accent rating, particularly when focused on suprasegmentals (Derwing and Rossiter 2003; Moyer 1999). Oral mimicry ability has also been identified in a number of previous L2 accent studies as a predictor of L2 foreign accent (Flege et al. 2001). In the study by Thompson (1991), ability to mimic was the third strongest predictor of foreign accent and the second strongest in Purcell and Suter (1980). If we consider HSK proficiency level as a rough gage of both pronunciation training and oral mimicry ability, the present findings support previous research into degree of L2 accent.

\subsection{Amount of formal instruction}

Strongly correlated with HSK proficiency level was the amount of formal Mandarin instruction (0.508), which had the third strongest correlation with the mean accent rating score (0.339). This result indicates that the amount of instruction affects the degree of L2 accent; however, not all previous L2 accent studies have shown the same results (Derwing and Munro 2013). This could be because instruction in other L2s does not typically include a large amount of pronunciation training compared to Chinese instruction (Munro and Derwing, 2011), where substantial pronunciation training is provided to help learners learn tones, as discussed. Given the degree that HSK scores are skewed toward pronunciation accuracy (see "Discussion" above), the results of the current study suggest that instruction focused on pronunciation can indeed improve accent, a fairly novel finding in the Chinese context.

\subsection{Motivational factors}

The final variable to enter the regression model was motivation, with a medium correlation with mean accent rating. For this variable, participants could select any of personal, academic, and professional reasons for learning Mandarin, from

2 As pointed out by an anonymous reviewer, the HSK proficiency level might not be a reliable indicator, because the HSK speaking test is administered separately to the main examination, which covers reading, writing, and listening. This study recorded proficiency level obtained in the general HSK test, and data are not available as to whether all the participants have the corresponding proficiency level in the HSK speaking test. 
none to all three. Results showed that participants learning Mandarin for multiple reasons had better accent scores, particularly when professional motivation was involved. These findings are supported by Moyer (2007: 514), who reported that closer-to-native performance was influenced by the speaker's intentions toward the language, "incorporating both professional and personal realms." Similar results were also obtained in an earlier study by Moyer (1999), where professional motivation in particular had a strong effect on accent rating.

Another motivation-related factor, the importance of sounding like an NS, also had a medium correlation with accent rating. For this variable, participants selected importance from 1 (not important) to 4 (very important); the mean score for all participants was 2.9 , with only 2 participants choosing "not important". These findings are supported by some previous L2 accent studies; for example, Purcell and Suter (1980) found that the strength of participants' concern for pronunciation accuracy was the fourth strongest predictor variable of actual accuracy, while Moyer (2007) found that desire to improve accent was positively correlated with accent ratings, as were participants' reasons for learning the L2. Interestingly, the importance of sounding like an NS also showed a small-to-medium negative relationship with LOR in this study; this is opposite to the results of Purcell and Suter (1980), where speakers' concern for pronunciation accuracy only significantly affected accent when combined with LOR. It is also worth mentioning that there was some variation in scores between L1 groups; for example, L1 Koreans $(n=5)$ were more concerned with sounding like NSs compared to other L1 groups, with a mean of 3.6. This could, in part, be explained by considering the Chinese context: most Chinese cities, including Nanjing, are less ethnically diverse compared to Western cities, where most previous L2 accent studies have occurred. In Nanjing, non-East Asian foreigners can normally be identified as foreign based simply on their physical appearance. The Korean participants may be keener to sound like NSs, because they do not "stand out" as foreigners in China, unlike the majority of the other participants in the current study.

Overall, the current results revealed that both speakers' desire to sound like an NS and their reasons for learning Mandarin affected their degree of L2 accent. These results reflect the findings from various other L2 accent studies, adding further support to the importance of motivation in SLA. As Piske et al. (2001: 202) noted, "most studies examining the effect of motivation on degree of L2 foreign accent have reported at least some influence of motivation on the outcome measures."

\subsection{Music}

Formal musical instruction had a medium correlation with accent scores. A total of 25 speakers had received an average of 3.5 years of musical instruction. As discussed, a link has been established between musical ability and L2 phonological ability, especially in tonal languages (Flege et al. 1995; Tahta et al. 1981; Thompson 1991). However, these studies have focused only on tone perception; no study 
has until now established a significant link between musical ability and degree of L2 accent. The present study is therefore likely the first to show a significant correlation between musical ability and degree of L2 accent and the only one to consider musical ability's effect on L2 Mandarin accent. Clearly, more research is needed.

\subsection{Sex}

Although there was no difference in mean accent scores between female and male participants, there were marked differences in correlations between accent scores and independent variables when bivariate correlations were conducted with a split dataset. For females, the amount of instruction had a strong positive correlation with accent scores, whereas for males there was no significant correlation. Selfrating of accent showed a strong correlation for females but only medium for males. This means that female participants were better able to judge the strength of their foreign accent than males. Another big difference was that motivational factors were significant predictors of female participants' accent scores, but not for male participants. For example, the importance of sounding like an NS and motivation showed medium-to-strong correlations with accent rating for females, but only weak ones for males. Overall, females' accent scores were more correlated with motivational factors than males' accent scores. These findings are similar to those of Moyer (2007), where a link was found between females' improvement in accent and positive attitudes, and of Thompson (1991) where females were more concerned with their pronunciation. Interestingly, the opposite was found in Flege et al. (1995), where motivational factors were significant predictors of L2 accent only for males. More recently, Polat (2011) discovered that "introjection orientation (because of obligation, avoidance of guilt or anxiety) was a low but significant negative predictor of accent native-likeness" for boys, but not girls (Polat 2011: 30). These past research findings, together with the current results, suggest that the effect of motivation on L2 accent could be subject to sex differences, but not conclusively - more research is needed.

\section{Limitations}

A limitation of this study is the number of speaker participants $(\mathrm{N}=70)$. Results would be more meaningful with a larger number of participants in proportion to the number of independent variables, particularly with regard to the regression analysis. A lower sample size reduces the sensitivity of the test, and therefore the results of the regression analysis are more conservative than they might otherwise be with a larger sample size. In multiple regression analysis, the ratio of participants to independent variables is disputed: Stevens (in Pallant 2010) suggested 15 participants per predictor, whereas Tabachnik and Fidell (2013) suggested 40 participants per predictor for stepwise regression. The current study included 8 variables in the regression analysis, which meant that there were 8.75 participants per predictor. However, despite not having more participants, stepwise multiple regression was still considered the best choice of analysis for the current 
study for the reasons outlined in section 3.4.3. Stepwise regression analysis has also been used in a number of previous L2 accent studies (Purcell and Suter 1980; Thompson 1991; Flege et al. 1995), thus making results more comparable to past findings.

The quantitative approach used in this study could also be considered a limitation. Although the majority of previous L2 accent studies have taken a quantitative approach, a mixed-methods approach may in fact have provided an understanding of how listeners judged accentedness of L2 Mandarin. One listener actually made notes next to some accent rating scores, which would have been interesting to discuss. For example, she wrote "sounds confident" next to a high-rated accent and "difficult to understand" next to a low-rated accent. If all listeners had been asked to make notes next to their rating scores as to why they assigned each score, these notes could have been analyzed qualitatively, like in the study by Yang (2016). A qualitative approach could have brought insight into how native Mandarin listeners judged the degree of accentedness and led to a better understanding of the concepts of accentedness and comprehensibility in L2 Mandarin.

\section{Conclusion}

This study explored which nonlinguistic factors affect the degree of foreign accent in L2 Mandarin. The results provide evidence that oral mimicry ability, pronunciation training, and motivational factors, and perhaps also musical ability, affect the degree of L2 accent and suggest sex differences in motivational factors affecting L2 accent. The most important finding was that self-rating of foreign accent was the best predictor of mean accent rating, followed by HSK proficiency level and then motivation. These findings are relevant to research into both L2 pronunciation and Chinese as an L2 and also have wider implications for L2 acquisition research in general.

These results point to the need for SLA research be more inclusive of non-European languages and in particular to consider that concepts such as accentedness and comprehensibility may not apply to lexical tone languages in the same way as other languages; the same may be true of languages characterized by other specific features. The fact that participants in this study could assess their own degree of foreign accent with such accuracy supports the idea that accentedness and comprehensibility are intertwined in Mandarin, probably because, since Mandarin is a tonal language, pronunciation errors are more likely to result in miscomprehension than in nontonal languages. Thus, although SLA research typically views accentedness and comprehensibility as related but distinct concepts, the results of the present study suggest that this view may be inaccurate for at least some L2s. This has broad implications for the field, whose theories and commonly held beliefs about language learning and L2 pronunciation may not always be cross-linguistically generalizable. 


\section{References}

Bergeron, Annie \& Pavel Trofimovich. 2017. Linguistic dimensions of accentedness and comprehensibility: Exploring task and listener effects in second language French. Foreign Language Annals 50(3). 547-566.

Bowles, Anita R., Charles B. Chang \& Valerie P. Karuzis. 2016. Pitch ability as an aptitude for tone learning. Language Learning 66(4). 774-808.

China Education Centre Ltd. 2016. HSK Speaking Test. http://www.chinaeducenter. com/en/hsk/hskadvanced.php (accessed 21 December 2017).

Derwing, Tracey M. \& Murray J. Munro. 1997. Accent, intelligibility and comprehensibility: Evidence from four L1s. Studies in Second Language Acquisition 20. 1-16.

Derwing, Tracey M. \& Murray J. Munro. 2013. The development of L2 oral skills in two L1 groups: A 7-year study. Language Learning 63(2). 163-185.

Derwing, Tracey M. \& Marian J. Rossiter. 2003. The effects of pronunciation instruction on the accuracy, fluency, and complexity of L2 accented speech. Applied Language Learning 13(1). 1-17.

Flege, James, Elaine M. Frieda \& Takeshi Nozawa. 1997. Amount of native-language (L1) use affects the pronunciation of an L2. Journal of Phonetics 25(2). 169-186.

Flege, James \& Serena Liu. 2001. The effect of experience on adults' acquisition of a second language. Studies in Second Language Acquisition 23(4). 527-522.

Flege, James, Murray J. Munro \& Ian, R. A. MacKay. 1995. Factors affecting strength of perceived foreign accent in a second language. Acoustical Society of America 97(5). 3125-3134.

Godfroid, Aline, Chin-His Lin \& Catherine Ryu. 2017. Hearing and seeing tone through color: An efficacy study of web-based, multimodal Chinese tone perception training. Language Learning 67(4). 819-857.

Guion, Susan, James E. Flege \& Jonathan D. Loftin. 2000. The effect of L1 use on pronunciation in Quichua-Spanish bilinguals. Journal of Phonetics 28. 27-42.

Hao, Yen-Chen. 2012. Second language acquisition of Mandarin Chinese tones by tonal and non-tonal language speakers. Journal of Phonetics 40(2). 269-279.

Hao, Yen-Chen. 2018. Contextual effect in second language perception and production of Mandarin tones. Speech Communication 97. 32-42.

Hosmer, David W. \& Stanley Lemeshow. 2000. Applied logistic regression. New Jersey: John Wiley and Sons, Inc.

Jesney, Karen. 2004. The use of global foreign accent rating in studies of L2 acquisition. Calgary, AB: Language Research Centre, University of Calgary.

Jiang, Xiaoli \& Andrew D. Cohen. 2018. Learner strategies for dealing with pronunciation issues in Mandarin. System 76. 25-37.

Kang, Okim. 2010. Relative salience of suprasegmental features on judgments of L2 comprehensibility and accentedness. System 38(2). 301-315.

Kinsella, Ciara \& David Singleton. 2014. Much more than age. Applied Linguistics 35(4). 441-462. 
Levins, John M. 2016. Accent in second language pronunciation research and teaching. Journal of Second Language Pronunciation 2(2). 153-159.

MacKay, Ian. R. A., James E. Flege \& Satomi Imai. 2006. Evaluating the effects of chronological age and sentence duration on degree of perceived foreign accent. Applied Psycholinguistics 27(2). 157-183.

Major, Roy C. 1986. Paragoge and degree of foreign accent in Brazilian English. Second Language Research 2(1). 53-71.

Marian, Victoria, Henrike K. Blumenfeld \& Margarita Kaushanskaya. 2007. The language experience and proficiency questionnaire (LEAP-Q): Assessing language profiles in bilinguals and multilinguals. Journal of Speech, Language, and Hearing Research 50(4). 940-967.

Moyer, Alene. 1999. Ultimate attainment in L2 phonology: The critical factors of age, motivation, and instruction. Studies in Second Language Acquisition 21. 81-108.

Moyer, Alene. 2007. Do language attitudes determine accent? A study of bilinguals in the USA. Journal of Multilingual and Multicultural Development 28(6). 502-518.

Moyer, Alene. 2013. Foreign accent: The phenomenon of non-native speech. Cambridge: Cambridge University Press.

Moyer, Alene. 2016. The puzzle of gender effects in L2 phonology. Journal of Second Language Pronunciation 2(1). 8-28.

Munro, Murray J. \& Tracey M. Derwing. 1998. The effects of speaking rate on listener evaluation of native and foreign-accented speech. Language Learning 48(2). 159-182.

Munro, Murray J. \& Tracey M. Derwing. 1999. Foreign accent, comprehensibility, and intelligibility in the speech of second language learners. Language learning 49(1). 285-310.

Munro, Murray J. \& Tracey M. Derwing. 2011. The foundations of accent and intelligibility in pronunciation research. Language Teaching 44(3). 316-327.

Pallant, Julie. 2010. SPSS Survival Manual. United Kingdom: Open University Press.

Penfield, Wilder \& Lamar Roberts. 1959. Speech and brain-mechanisms. Princeton, NJ: Princeton University Press.

Piske, Thorsten, Ian R. A. MacKay \& James E. Flege. 2001. Factors affecting degree of foreign accent in an L2: A review. Journal of Phonetics 29(2). 191-215.

Plonsky, Luke (ed.). 2015. Advancing quantitative methods in second language research. New York: Routledge.

Plonsky, Luke \& Frederick L. Oswald. 2014. How big is "big”? Interpreting effect sizes in L2 research. Language Learning 64(4). 878-912.

Polat, Nihat. 2011. Gender differences in motivation and L2 accent attainment: An investigation of young Kurdish learners of Turkish. Language Learning Journal 39(1). 19-41.

Polat, Nihat \& Diane L. Schallert. 2013. Kurdish adolescents acquiring Turkish: Their self-determined motivation and identification with L1 and L2 communities as predictors of L2 accent attainment. The Modern Language Journal 97(3). 745-763. 
Purcell, Edward T. \& Richard W. Suter. 1980. Predictors of pronunciation accuracy: A re-examination. Language Learning 30(2). 271-287.

Saito, Kazuya. 2015. Experience effects on the development of late second language learners' oral proficiency. Language Learning 65(3). 563-595.

Saito, Kazuya, Pavel Trofimovich \& Talia Isaacs. 2015. Using listener judgements to investigate linguistic influences on L2 comprehensibility and accentedness: A validation and generalisation study. Applied Linguistics 36(4). 1-25.

Saito, Kazuya, Pavel Trofimovich \& Talia Isaacs. 2016. Second language speech production: Investigating linguistic correlates of comprehensibility and accentedness for learners at different ability levels. Applied Psycholinguistics 37(2). 217-240.

Sereno, Joan, Lynne Lammers \& Allard Jongman. 2016. The relative contribution of segments and intonation to the perception of foreign-accented speech. Applied Psycholinguistics 37(2). 303-322.

Slevc, L. Robert \& Akira Miyake. 2006. Individual differences in second-language proficiency: Does musical ability matter? Psychological Science 17(8). 675-681.

So, Connie K \& Catherine T. Best. 2010. Cross-language perception of non-native tonal contrasts: Effects of native phonological and phonetic influences. Language and Speech 53. 273-293.

Sun, Chaofen. 2006. Chinese: A linguistic introduction. Cambridge: Cambridge University Press.

Suter, Richard W. 1976. Predictors of pronunciation accuracy in second language learning. Language Learning 26(2). 233-253.

Tabachnick, Barbara G. \& Linda S. Fidell. 2013. Using multivariate statistics, 6th edn. Boston, MA \& London: Pearson.

Tahta, Sonia, Margaret Wood \& Kate Loewenthal. 1981. Foreign accents: Factors relating to transfer of accent from the first language to a second language. Language and Speech 24(3). 265-272.

Thompson, Irene. 1991. Foreign accents revisited: The English pronunciation of Russian immigrants. Language Learning 41(2). 177-204.

Trofimovich, Pavel \& Talia Isaacs. 2012. Disentangling accent from comprehensibility. Bilingualism: Language and Cognition 15(4). 905-916.

Trofimovich, Pavel, Talia Isaacs, Sara Kennedy, Kazuya Saito \& Dustin Crowther. 2016. Flawed self-assessment: Investigating self- and other-perception of second language speech. Bilingualism: Language and Cognition 19(1). 122-140.

Uzal, Melike, Teemu Peltonen, Minna Huotilainen \& Olli Aaltonen. 2015. Degree of perceived accent in Finnish as a second language for Turkish children born in Finland. Language Learning 65(3). 477-503.

Wang, Xinchun. 2013. Perception of Mandarin Tones: The effect of L1 background and training. The Modern Language Journal 97(1). 144-160.

Wayland, Ratree. 1997. Non-native production of Thai: Acoustic measurements and accentedness ratings. Applied Linguistics 18(3). 345-373. 
Yang, Bei. 2012. The gap between perception and production of tones by American learners of Mandarin - An intralingual perspective. Chinese as a Second Language Research 1(1). 33-53.

Yang, Chunsheng. 2016. The acquisition of L2 Mandarin prosody: From experimental studies to pedagogical practice. Philadelphia \& Amsterdam: John Benjamins Publishing Company.

Zhao, Yang. 2011. A tree in the wood: A review of research on L2 Chinese acquisition. Second Language Research 27(4). 559-572.

Zybert, Jerzy \& Sabina Stepien. 2009. Musical intelligence and foreign language learning. Research in Language 7. 99-111.

\section{Appendix A - Materials}

wǒ qùnián qiūtiān láidào zhōngguó zài nánjīng dàxué xué xí hànyǔ wǒ měi zhōu yǒu 我去年秋天來到中國, 在南京大學學習漢語。我每周有 èr shí sì jié zhōngwén kè xià kè hòu wǒ zuì xỉhuan hé péngyǒu qùjiǔbā hé bùtóng guójiā 二十四節 中 文課。下課後, 我最喜歡和 朋友 去酒吧。和不同國家 de rén yì qĩ jiāoliú néng liǎojiě hěnduō yǒuqùde shìqing zhè ràng wǒ zēngzhăng le bù 的人一起交流, 能了解很多有趣的事情, 這讓我增長了不 shăo jiànshi fàngjià de shíhòu wǒ huì qù lǔ yóu zhè kě yǐ ràng wǒ gèng liǎojiě 少見識。放假的時候, 我會去旅遊, 這可以讓我更了解 zhōngguó 中國。

Lani Freeborn

Mailing address: English Language Teaching Unit, The Chinese University of Hong Kong Room 434, Li Dak Sum Building, Shatin, Hong Kong

Email: Iani.freeborn@cuhk.edu.hk

John Rogers

Mailing address: The Education University of Hong Kong, 10 Lo Ping Road, Tai Po, New Territories, Hong Kong

Email: $\quad$ rjrogers@eduhk.hk

Received: June 7, 2018

Accepted: $\quad$ November 21, 2018 


\title{
影響第二語言普通話外國口音程度的非語言學因素
}

\author{
Lani Freeborn ${ }^{1}$, John Rogers ${ }^{2}$ \\ 香港中文大學 $^{1}$ 、香港教育大學 ${ }^{2}$
}

\section{摘要}

先前的研究發現有一些非語言學因素會影響到第二語言學習時產生的所能感知到的 外國口音強度。然而, 過去的大多數研究主要關注印歐語系, 特別是英語的外國口 音。因此, 那些在其他語言中影響外國口音的因素是否同樣影響普通話, 尚未可知。 本文將報告一項以普通話為第二語言, 關注非語言學因素對外國口音影響的研究結 果。70 位普通話漢語學習者錄下了語音樣本, 並完成了語言背景調查問卷。由 15 位普通話母語者根據一個 1-9 分外國口音程度李克特量表, 對語音樣本進行打分。 逐步多元回歸分析得到了一個發音準確性的三因數預測模型：外國口音的自評, 漢 語水平考試的能力水平, 動機理由。研究結果表明 : 1) 在普通話二語學習中, 影響 外國口音的因素可能與先前二語口音研究所發現因素有所不同；2) 與非聲調語言 相比, 在如同普通話這樣的聲調語言中, 口音和可理解性兩者間的關聯更為複雜。 本研究的發現在被英語二語學習主導的第二語言習得的研究領域中有更為廣泛 的應用。

\section{闒鍵䓂}

外國口音, 第二語言, 普通話, 可理解性 